\title{
Exposición al plomo y sus efectos en la salud infantil
}

\author{
M.C. Jeanette Vega $\mathbf{M}^{1}$; M.C. Adela Contreras $R^{2}$; M.C. Etnesto Ríos $\mathrm{L}^{3}$; \\ Ing. Nella Marchetti P.4; Milena Agurto $V^{5}$

\section{Cronic lead exposure and its effects in child health}

\begin{abstract}
Chtldhood lead poisoning is a man-made disezse whose magnitude has not yet been measured in Chile. In the U.S.A. it has been estimated that 4 percent of children have elevated blood lead levels and that among poot black children, this rate is as high as 18 percent. The main sources of symptomatic lead poisoning are lead-based paint used in interior and exterior house surfaces, airbone lead from leaded gasoline and industrial emissions. Lead can be transferred from any source to soil and dust. Other sources of lead are contamined food and water, occupational sources, and lead glazed pottery. Neurological symptoms (hyperactivity, distractibility, lower intellectual development), psychological difficulties (behavioral deficits), hematologic abnormalities (reduction in the biosyntesis of heme, anemia), and metabolic changes (reduction in concentration of $1-25$ dihydroxy vitamin $D$ and in the metabolism of erythrocyte pyrimidine) have been shown to occur in lead poisoning. Recent data suggests that prenatal exposure to lead my be related to minor congenital abnormalities, tumors of the kidney, and growth abnormalities.

(Key words: lead poisoning, chronic lead exposure, elevated blood lead level.)
\end{abstract}

La interacción de la especie humana con su medio ambiente produce múltiples efectos, algunos benéficos y otros dañinos.

El desartolio industrial ha traído aparejada la acumulación de distintos elementos potencialmente tóxicos para el organismo, entre éstos el plomo, que se encuentra en el medio ambiente principalmente como resultado de dicha actividad y no tiene hasta el momento una función fisiológica conocida, pero sí efectos adversos para la salud humana.

\section{Fuentes de plomo}

Aire. El plomo del aire proviene principalmente de las emisiones producidas por los vehículos de locomoción que utilizan gasolina plomada. En Chile, el plomo se utiliza en la gasolina como elemento antidetonante. La gasolina actualmente en

1. Docente, Departamento de Salud Pública, Facultad de Medicina, Universidad de Chile, Campus Oriente.

2. Becado, Departamento de Salud Pública, Facultad de Medicina, Universidad de Chile, Campus Oriente.

3. Servicio de Hematologia, Hospital Luis Calvo Mackenna.

4. Ingenjero, Departamento de Salud Ocupacional y Contaminación Ambiental, Instituto de Salud Pública.

5. Ayudante alumr.o, Departamento de Salud Pública, Facultad de Medicina, Campus Oriente, Universidad de Chile. uso contiene aproximadamente $1,5 \mathrm{~g}$ de $\mathrm{Pb} / \mathrm{C} \mathrm{Co}$ municación personal Sr. J. Navarro, ingeniero de ENAP). Esto se traduce en que cada automóvil lanza al aire aproximadamente $60 \mathrm{~g}$ de plomo por cada 40 litros de gasolina, lo cual, si lo extrapolamos al parque automotriz existente en Santiago ( 400.000 vehículos) da una cifra de 1.250 toneladas de plomo emitidas por ano a la atmósfera ${ }^{1}$, sin considerar los motores Diesel.

La gasolina sin plomo ha sido introducida en Europa y los Estados Unidos hace aproximadamente 10 años; esto ha tra ido aparejada una disminución importante en los niveles de plomo en el aire ${ }^{2}$.

El plomo del aire es absorbido por el ser humano por inhalación e ingestión de polvo o tierra contaminada con plomo, to que se produce principalmente en los niños. Estos están más expuestos por dos razones: la ingestión permanente de polvo y a que, debido a su menor estatura, están más cerca de la tierra, facilitándose la inhalación de polvo contaminado. Cada vez que el niro se lleva los dedos a la boca o ingiere un alimento que se ha caído al suelo, está ingiriendo algunos microgramos de plomo.

Los nifios que viven cerca de algunas fuentes de emisión, como fábricas de bater ías y fundiciones, están particularmente expuestos.

Alimentos. Con la dieta normal se ingieren aproximadamente 150 microgramos de plomo al día, provenientes de la contaminación ambiental de 
los alimentos, 10\% de los cuales son absorbidos y retenidos en los adultos y $45 \%$ a $60 \%$ en los niños ${ }^{3}$.

Pintura. Otra fuente importante de exposición al plomo son las pinturas, siendo ésta una causa frecuente de intoxicación clínica en los niños ${ }^{3}$. Hasta el año 1940 la pintura utilizada en E.U.A. contenía plomo. Actualmente, sin embargo, sólo la pintura utilizada en superficies exteriores puede contener una cantidad no mayor de $0,06 \%$ $(600 \mathrm{ppm})$ de plomo por peso seco. El plomo de esta fuente es inhalado o ingerido principalmente aI resquebrajarse las pinturas, lo cual produce gran cantidad de partículas de plomo flotando en el ambiente. $\mathrm{El}$ riesgo de ingestión $e$ inhalación es, por lo tanto, mayor en las casas antiguas o en vías de reparación, pues estas últimas requieren, habitualmente, remover la pintura antigua, lo cual a su vez genera un aumento importante del plomo en el aire y polvo ambiental. En Chile no existen normas que regulen el contenido de plomo de las pinturas y en general prácticamente todas tienen plomo en distintas cantidades de acuerdo al tipo y color (Comunicación personal Sr. N. Carter, químico de empresas pinturas Tricolor, Santiago).

Agua. La existencia de plomo en el agua está relacionada con el uso de canterías de plomo y sol. daduras. El agua de ph ácido aumenta la posibilidad de que partículas de plomo sean liberadas al torrente 4 .

Fuentes ocupacionales. El plomo puede impregnar la piel, el cabello, los zapatos, la ropa y los vehículos de trabajadores expuestos en su ambiente laboral y de este modo ser trasladado desde el sitio de trabajo a las casas.

Existen algunos estudios que han demostrado que los hijos de los trabajadores expuestos tienen concentraciones sanguíneas de plomo elevadas ${ }^{5}$.

Otras fuentes. Otras fuentes de plomo han sido descritas, por ejemplo, en las cerámicas que contienen barnices plomados, algunos alimentos como la merhuza ${ }^{6}$ y ciertas comidas en conserva ${ }^{7}$, especialmente los de $\mathrm{pH}$ ácido, que aumenta la probabilidad de desprendimiento de partículas de plomo.

Dadas estas múltiples fuentes, los nifios de todos los países tienen plomo depositado en su organismo. Las cantidades son diferentes en cada país, dependiendo de las condiciones en cuanto a posibles fuentes y a la existencia de normas de control de éstas. Una medición muy usada es la tasa de prevalencia de concentraciones altas de plorno sanguíneo en nifíos menores de seis aflos. En E.U.A. se calcula que 3\% de los niños tienen concentraciones altas, con tasas fluctuantes entre 2\%, en niños blancos, caucásicos, de clase media, y 18\% en nifnos negros de bajo nivel socjoeconómico ${ }^{2}$. En Europa los promedios de concentraciones sanguineas de plomo fluctúan entre $9 \mu \mathrm{g} / \mathrm{dl}$ en Suecia y $29 \mu \mathrm{g} / \mathrm{dl}$ en Finlandia, población urbana ${ }^{8}$. En Chile esta tasa no ha sido medida.

Un diagrama de las fuentes posibles de plomo en el ambiente del nif́o se muestra en la figura.

\section{Efectos del plomo en ta salud infantil}

El plomo se distribuye en el organismo primariamente en dos compartimientos, uno activo en la sangre $y$ tejidos blandos y otro pasivo o de depósito en los huesos. Con la edad, el contenido de plomo en los huesos aumenta significativa. mente.

En la última década el conocimiento acerca de la toxjcidad del plomo en niños ha ido creciendo, otorgándose importancia a concentraciones sanguíneas cada vez menores como causas de daños.

Estudios epidemiológicos cuidadosos en Escocia, Dinamarca y Grecia ${ }^{10}$ han demostrado alteraciones en nifos con concentraciones sanguineas tan bajas como 10 a $15 \mu \mathrm{g} / \mathrm{dl}$. Estos estudios han identificado y controlado las variables no relacionadas con el plomo, que pueden actuar como potenciales factores confundientes. Un documento

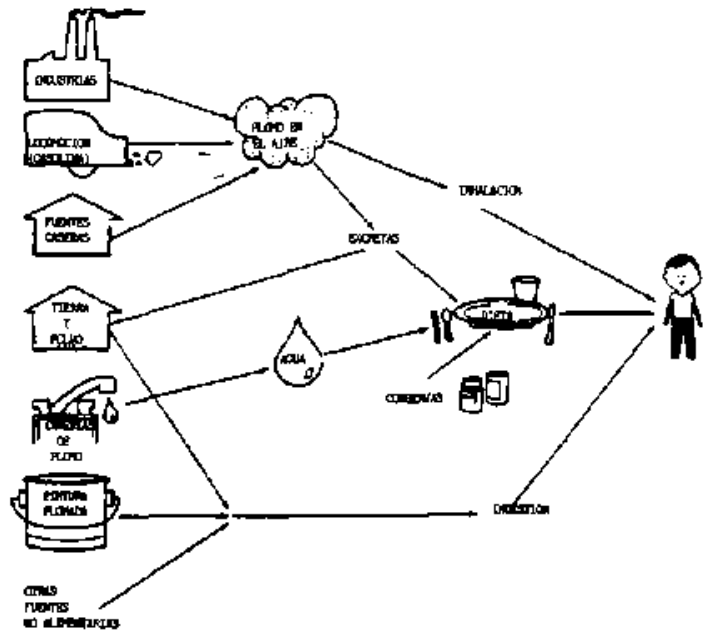

Figura: Fuentes de plomo en el ambiente del niño. 
reciente de la Agencia para la Protección Ambiental de E.U.A. ha sugerido que 10 a $15 \mu \mathrm{g} / \mathrm{d} 1$ constituyen una concentración de plomo suficiente para producir alteraciones neuropsicológicas ${ }^{11}$.

Estudios recientes documentan la asociación entre plomo y reducción de la síntesis de grupo $\mathrm{Hem}^{12}$, disminución de la concentración de 1,25 dihidroxicolecalciferol (vitamina $D)^{13}$ y alteraciones en el metabolismo de la pirimidina eritrocitaria $^{14}$.

Además, se ha demostrado que la exposición crónica a altas concentraciones de plomo se asocia a alteraciones neurofisiológicas, electroencefa$\operatorname{lográficas~}^{15}$ y disminución en la velocidad de conducción nerviosa ${ }^{16}$.

La toxicidad del plomo es evidente en los glóbulos rojos y sus precursores, los rifiones y los sistemas nerviosos centra` y periférico. Nuevos datos $^{17}$ sugieren que la exposición prenatal a pequeñas cantidades de plomo se relacionaría con anormalidades congénitas menores y en animales, con tumores renales.

Otros estudios, realizados entre los antos 1985 y 1988, comprueban los diversos efectos tóxicos del plomo, principalmente en la esfera neurológi$\mathrm{ca}^{18}$, hematológica ${ }^{19}$ y psicológica ${ }^{20}$. En monos, la administración de plomo desde el nacimiento, durante 100 dias, produce, tanto en los ejemplares jóvenes y adultos, que habian sido expuestos a concentraciones altas y moderadas, déficit estadísticamente significativos en conductas de discriminación con respecto a los controles, que correspond ian claramente a disturbios de la aten$\operatorname{ción}^{2 t}$.

Los primeros estudios experimentales fueron efectuados en $1979^{22}$ y repetidos posteriormente en Inglaterra y Grecia ${ }^{10}$. Muchos de ellos se han referido a los efectos en la inteligencia, percepción y aprendizaje. Se ha especulado, con pocos datos, sobre el efecto del plomo en algunas conductas sociales complejas del individuo. En casos de exposición al plomo se ha demostrado mayor incidencia de conductas asociadas al síndrome de déficit de la atención entre los nifios expuestos ${ }^{22}$. El déficit de la atención y los trastornos de aprendizaje son factores conocidos de riesgo de conductas antisociales. La tasa de delincuencia en niños que presentan déficit de la atención y trastornos de la conducta en la infancia es de 0,58 . EJ riesgo atribuible de hiperactividad en nifos con niveles de plomo elevados es de 0,55. Multiplicando estas dos cifras obtendríamos una proba- bilidad de 0,20 de conductas antisociales en los individuos con exceso de exposición al plomo. La existencia de una relación causal entre plomo y delincuencia no ha sido estudiada, pero estas cifras permiten hacer conjeturas al respecto ${ }^{10}$.

Los efectos tóxicos del plomo no se limitan al sistema nervioso. La exposición intrauterína $y$ temprana al plomo en dosis bajas interfiere en el crecimiento del niño en el primer año de vida. Las concentraciones de plomo son inversamente proporcionales a la altura y circunferencia toráci$\mathrm{ca}^{23}$. También se han encontrado defectos de la audición en asociación con concentraciones elevadas de plomo en sangre ${ }^{24}$.

Los sintomas de intoxicación aguda por plomo son: fatiga, anorexia, palidez, astenia, irritabilidad, alteraciones del sueño, cambios bruscos de conducta y retardo mental. Las manifestaciones más serias son torpeza motora, ataxia, dolor abdominal, vómitos, constipación y alteraciones de conciencia debido a encefalopatía.

El plomo, una vez absorbido (por inhalación, ingestión o vía percutánea), se distribuye en los tejidos blandos del organismo y huesos. Las concentraciones sanguíneas reflejan el equilibrio dinámico entre absorción, excreción y depósito ${ }^{25}$.

En el niño, el tipo de nutrición es un factor significativo de riesgo. La dieta rica en grasa, po. bre en calcio ${ }^{26}$ o hierro ${ }^{27}$, aumentarían la absorción de plomo por el intestino. Las deficiencias de hierro, calcio y fósforo muestran correlaciones positivas con concentraciones altas de plomo en humanos y animales experimentales ${ }^{28}$.

En consideración a la magnitud del problema, en E.U.A. se han utilizado desde hace una década métodos de rastreo para la detección de los niffos con concentraciones altas de plomo. La mejor manera de determinar la exposición al plomo es a través de sistemas de vigilancia biológica como determinaciones de protoporfirina eritrocitaria (PPE) y concentración de plomo en la sangre.

El propósito de los programas de rastreo es identificar los nif̃os con exposición importante al plomo en forma precoz, de modo de prevenir las consecuencias de la toxicidad del plomo. Los programas de rastreo son diferentes de los métodos de diagnóstico, porque se efectúan en sujetos asintomáticos. Los nifros con concentraciones altas son tratados, de acuerdo a la severidad de la acumulación de $\mathrm{Pb}$, en forma más o menos agre. siva. Además, se efectúa un manejo ambiental de los casos, investigando las fuentes probables de plomo para luego aplicar las medidas destinadas a 
controlar la exposición (por ejemplo, removiendo la pintura de la casa).

Las concentraciones elevadas de plomo son más peligrosas en los niños entre nueve meses y tres años de edad. Estos tienen, por lo tanto, prioridad en los programas de rastreo. Se recomienda que el programa esté incluido en el de Control de Niño Sano, realizando el primer examen entre los nueve y doce meses de edad y pos. teriormente cada año hasta los seis años de edad.

Los exámenes más utilizados son las mediciones de protoporfitina eritrocitaria (PPE) y de plo. mo en sange capilar o venosa. Dada la mayor facilidad de recolección, rapidez y sencillez del procedimiento, la recolección en sangre capilar es el método de elección ${ }^{29}$.

En Chile se efectúan únicamente mediciones de protoporfirina y plomo en sangre venosa. Sólo en un laboratorio se ha iniciado recientemente la determinación de zinc-protoporfirina utilizando sangre capilar, en trabajadores expuestos al plomo. Tampoco se utiliza la medición de la concentración de plomo en sangre capilar, para lo cual es necesario contar con un detector de trazas de metales.

Una ventaja del rastreo de protoporfirina en sangre venosa o capilar es que cuanto ésta se encuentra elevada en niños no expuestos al plomo, usualmente refleja deficiencia de hierro ${ }^{30}$. Los primeros signos de deficiencia de hierro son anormalidades bioquímicas (bajas concentraciones séricas de ferritina, aumento de la PPE), seguidas de anormalidades celulares (microcitosis e hipocromía). El ensayo de PPE ha sido probado como método de rastreo para deficiencia de hierro en una población de 4.160 niños, habiéndose encontrado que el línite superior normal es $35 \mu \mathrm{g} / \mathrm{dl}$.

La deficiencia de hierro es común en muchos grupos en riesgo de intoxicación por plomo, especialmente niños de nivel socioeconómico bajo, que habitan casas antiguas y tienen entre 9 y 24 meses de edad. La más alta frecuencia de intoxicaciones por plomo se encuentra a los 36 meses. La deficiencia de hierro y la intoxicación por plomo pueden ocurrir en el mismo niño. Más aún, evidencias experimentales indican que la deficiencia de hierro aumenta la proporción de plomo absorbido desde el intestino y agrava los efectos tóxicos de éste ${ }^{31}$. Estudios de prevalencia en Chile han determinado que en el lactante se observa eritropoyesis deficiente en hierro, detectada por elevación de la protoporfirina eritrocitaria en 41 \% de la población y que esta carencia llega a su etapa final de anemia en $31 \%$ de ellos ${ }^{32}$. En preescolares con carencia de hierro se observa elevación de la protoporfírina eritrocitaria en $33 \%$ y anemia en $19 \%$.

Estos hechos justifican que, para separar la in. toxicación por plomo de la carencia de hiero, se estudien también parámetros relacionados al hierro cuando se está determinando la prevalencia de concentraciones sanguineas elevadas de plomo en los nifros.

La protoporfirina eritrocitaria y la concentración sanguinea de plomo miden distintos aspecto de la intoxicación por plomo. La PPE expresa la cantidad de protoporfírina que existe en los glóbulos rojos. Se trata de una molécula proteica que se une al hierro para formar el grupo Hem de la hemoglobina. La enzima mitocondrial que cataliza esta reacción en la médula ósea es la ferroquelatasa y las concentraciones altas de plomo la inhiben, aumentando, por lo tanto, la cantidad de protoporfirina libre, cuyas concentraciones altas reflejan, en consecuencia, los efectos metabólicos de] plomo en la médula ósea ${ }^{3}$. La concentración sanguinea de plomo, en cambio, refleja el grado de absorción; por lo tanto, es una medida de la exposición actual. Se considera positivo un valor mayor de $25 \mu \mathrm{g} / \mathrm{dl}$. Fluctuaciones en las concentraciones sanguíneas de plomo en períodos cortos pueden deberse a variaciones fisiológicas de la absorción o a exposiciones agudas esporádicas al metal.

\section{Interpretación de los resultados del rastreo}

Los niffos pueden ser arbitrariamente divididos en 4 clases de acuerdo a sus valores de protoporfirina y plomo sanguíneo. Esta clasificación, desarrollada por el Centro de Control de Enfermedades de E.U.A. (CDC), sugiere el riesgo relativo y la prioridad para evaluación médica e inter. vención ambiental (tabla).

Clase I. Bajo riesgo. No se requiere investigación ambiental para estos niffos.

Clase II. Riesgo moderado. Debe realizarse una investigación en las casas de estos nifios dentro de 10 djas a contar de la fecha de notificación de los exámenes.

Clase III. Alto riesgo. Debe realizarse una investigación ambiental dentro de los 5 días desde la notificación de los exámenes y los nif̂os deben ser tratados. 
Tabla

Clasificación del Centro de Control de Enfermedades de E. U.A. del riesgo en niños asintomáticos y priondad para evaluación médica.

\begin{tabular}{lcccc}
\hline Plomo en sangre & \multicolumn{4}{c}{ Protoporfirina eritrocitaria (ug/dl) } \\
\hline ug/d) & $<3-5$ & $35-74$ & $75-174$ & $>175$ \\
No realizado & I & $*$ & $*$ & $*$ \\
$<24$ & I & Ia & Ia & PPE(+) \\
$25-49$ & Ib & II & III & III \\
$50-69$ & $* *$ & III & III & IV \\
$>70$ & $* *$ & $* *$ & IV & IV \\
\hline
\end{tabular}

PPE $\left(^{+}\right)$: Protoporfirina eritropoy ética: rara vez la deficiencia de hierto puede producir valores de protoporfirina tan elevados.

* : Es necesario medir el plomo sanguíneo para estimar riesgo.

** : Combinación de resultados no observada en la práctica. Si aparecen, hay que corroborarlos inmediatamente con una muestra de sangre venosa.

Clase JV. Riesgo urgente. Debe realizarse una investigación ambiental dentro de las 48 horas siguientes y los niños deben ser tratados a la brevedad posible.

\section{Medidas de control en Chile}

En nuestro pais no existen normas de calidad del aire para plomo. Esto es particularmente grave, dado que la contaminación atmosférica ha alcanzado niveles alarmantes en los últimos afios, especialmente debido a partículas, dentro de las cuales están incluidas las respirables, una de ellas el plomo.

En E.U.A. ${ }^{33}$ la concentración máxima permitida es de 1,5 $\mu \mathrm{g} / \mathrm{m}^{3}$. En Santiago de Chile la concentración de particulas es determinada a través de dos redes de vigilancia. Una de ellas funciona desde 1977 y consta de 15 estaciones de muestreo, que determinan el nivel total de partículas. De acuerdo a datos suministrados por el Servicio de Salud Metropolitano del Ambiente, la estación que registró mayor concentración de partículas en los años 1988 y 1989 es la ubicada en el con. sultorio Carlos Avendaño del Servicio de Salud Metropolitano Occidente y la estación que registró menos partículas es la del consultorio $\mathbf{L a}$ Pintana, en el Servicio de Salud Metropolitano Sur-Oriente.
Existe una segunda red de vigilancia en funcionamiento desde 1988, la red MACAM. En ésta, se efectúan mediciones de la concentración de partículas totales y respirables $(<$ de 10 micrones), subdivididas en una fracción gruesa de 2,5 a 10 micrones y una fracción fina, $<$ de $2,5 \mathrm{mi}$ crones. Esta red consta de 4 estaciones fijas y una móvil.

La norma norteamericana para la concentración de particulas menores de 10 micrones (PM10) es $50 \mu \mathrm{g} / \mathrm{m}^{3}$ (estándar primario y secundario) y $150 \mu \mathrm{g} / \mathrm{m}^{3}$ (estándar primario y secundario) como cifra máxima aguda que no debe ser sobrepasada más de una vez cada año ${ }^{33}$. De acuerdo a los datos proporcionados por el Servicio de Salud Metropolitano del Ambiente, los valores promedio mensuales de todas las estaciones de la red MACAM superaron $\operatorname{los} 150 \mu \mathrm{g} / \mathrm{m}^{3}$ durante todo el año 1988 y el segundo semestre de 1989.

En cuanto a las concentraciones de plomo en el aire, mediciones efectuadas en el patio del Instituto de Salud Pública muestran promedios mensuales fluctuantes entre 0,7 y $1,73 \mu \mathrm{gg} / \mathrm{m}^{3}$, alcanzando valores tan altos como $3,2 \mu \mathrm{g} / \mathrm{m}^{3}$ en abril de 1989 (Comunicación personal Sr. Flavio Vega, ingeniero jefe laboratorio de Sajud Ocupacional, Instituto de Salud Pública).

En nuestro país no existen normas que regulen la concentración de plomo en pinturas, agua o alimentos y la gasolina que se emplea contiene plomo, como ya se ha mencionado. Recientemente se ha anunciado que a partir de 1990 se comenzará a utilizar progresivamenge gasolina sin plomo.

Por los múltiples efectos que la concentración sanguínea elevada de plomo produce en los ninos, el nivel crítico de contaminación atmostérica especialmente por partículas en Santiago, la no exjstencia de normas que regulen la concentración de plomo en el aire, las pinturas y los ali. mentos, y el uso en el pais de gasolina plomada, ser ia importante determinar las concentraciones sanguineas de plomo en una muestra representativa de la población infantil de Santiago para definir la magnitud del probletna.

\section{RESUMEN}

La intoxicación por plomo en los nifios es una enfermedad provocada por el hombre. En Chile la magnitud del problema no ha sido medida. En E.U.A. se ha estimado que aproximadamente 
$4 \%$ de los nifios tienen concentraciones sanguíneas de plomo elevadas, ascendiendo esta cifra a $18 \%$ de los nifios negros pobres. Las principales fuentes de plomo que causan intoxicación sintomática son las pinturas plomadas utilizadas en el interior y exterior de las casas; el aire contaminado debido al uso de gasolina plomada y emisiones industriales; la tierra y polvo a los que el plomo puede ser transferido desde cualquier fuente; los alimentos y el agua contaminada; barnices plomados usados en cerámicas y otras fuentes ocupacionales. El plomo produce efectos neurológicos (hiperactividad, trastomos de la atención, retardo del desarrollo intelectual), psicológicos (trastornos conductuales), hematológicos (reducción de la sintesis del grupo Hem, anemia), metabólicos (reducción en la concentración de 1 a $25 \mathrm{di}$. hidroxivitamina D y trastornos en el metabolis. mo de la pirimidina eritrocitaria). Nuevos datos sugieren que la exposición prenatal al plomo se podría relacionar con anormalidades congénitas menores, retardo del crecimiento y tumores renales.

(Palabras clave: intoxicación por plomo, exposición crónica al plomo, concentración sanguínea de plomo elevada.)

\section{AGRADECIMIENTOS}

Los autores desean expresar sus agradecinientos a la Dra. Gabriela Venturini R., Bioquimico Sra. Carmen Oyanguren e Intemo de Medicina Sr. Mariano Montenegro por la aycuda prestada en la preparación y revisión de este manusctito.

\section{REFERENCIAS}

1. Molina P.: Frente al Smog: "La solución exige queser pagarla". Diario "El Mercurio". Cuerpo D, Domingo 11 de junio, 1989. Págs. 1-6.

2. Preventing Lead Poisoning in Young Children. A Statement by the Centers for Disease Control. January 1985. U.S. Deporment of Healih and Human Services.

3. Piomelli $S$ : Lead Poisoning, its detection and treatment. Drug Theraphy (HOSPj 1977; 3: 19-32.

4. Morse D.L. Warson W.N., Houseworth, J. at al.: Exposure of children to lead in drinking water. Am J Public Health 1979; 69: 711-721.

5. Baker E.L. Jr., Folland D.S., Taylor T.A. et al.: Lead poisoning in children of lead workers: home contamination with industrial dust. $N$ Engl J Med 1977 ; 296: 260-261.

6. Diaz O.. Aguirre S., Clucci L.: Determinación de Cadmio y Plomo obtenido de San Antonio (Chile). Rev Chil Nut 1984; 12 : 121.

7. Diaz D., Compos P., Espinoza J. y Cols: Concen- tración de Cadmio y Plomo en harina de pescado y jurel en conserva. Rev Chil Nutr 1986; $14: 77$.

8. National Swedish Institute of Environmental Medicine and Department of Environmental Hygiene, Assessment of human exposure to lead and cadmium through biological monitoring. Karolinska Institute. Stockholm, 1982.

9. National Academy of Sciences (NAS), Committee on Toxicology. Recomendations for the prevention of lead poisoning in children. Washington, D.C.: National Research Council, July 1976.

10. Needleman H.L.: The persistent threat of lead: A singular opportunity. Am J Public Health 1989; 79: 643-645.

11. Davis J.M., Svendsgaard D.J.* Lead and child development. Nature 1987; $329: 297-300$.

12. Piomelli S., Rosen J.F., Chisoln J.J, et al: Management of childhood lead poisoning. J Pediatr 1984; 105: 523-532.

13. Mahaffey K.R. Rosen J.F., Chesney R.W., et al.: Association between age, blood lead concentration and serum 1,25-dihydroxycholecalciferol jevels in children. Am J Clin Nutr 1982; 35: 1327-1331.

14. Angle C.R., McIntire M.S: Low level and inhibition of erythrocyte pyrimidine nucleotidase. Environ Res 1978; 17: 296-302.

15. Otto D., Benignus V., Muller K., et al: Effect of low to moderate lead exposure on slow cortical potentials in young children: two-year follow-up study, Neurobehay Toxicol Teratol 1982; 4: 733737.

16. Seppalainen A.M., Hernberg, S.: A follow-up study of nerve conduction velocities in lead exposed workers. Neurobehav Toxicol Teratol 1982; 4: 721723.

17. Needleman H.L., Rabinowitz M., Leviton A., et al.: The relationship between prengtal exposure to lead and congenital anomalies. JAMA 1984; 251:322.

18. Schwortz J., Landrigan P.J., Feldman R.G. et al. Threshold effect in lead-induced peripheral neuropathy, United States Environmental Protection Agency, Washigton, DC. J Pediatr 1988; 112 : 12 17.

19. Piomelli $S$, Seaman $C$, Zullow $D$., ef at: Threshold for lead damage to heme synthesis in urban children. Proc Natl Acad Sci USA 1982; 79: 33353339 .

20. Foust D., Brown J.i Moderately eleveted blood lead levels: effects on neuropsychologic functioning in children. Rhode Island Hospital, Department of Psychiatry, Providence 02902. Pediatrics 1987; 80: 623-629.

21. Gilbert S.G., Rice D.C. Low level lifetime lead exposure produces behavioral toxicity (spatial discrimination reversal) in adult monkeys. Toxicology Research Division, Health and Welfare Canada, Ottawa. Toxicol Appl Pharmacol 1987; 91: 489490.

22. Needleman H.L., Gunnoe C, Leviton A., et al: Deficits in psychologic and classroom performance of children with elevated dentine lead levels. N Engl 3 Med 1979; 300: 689-695.

23. Schwartz J., Angle C., Pitcher H.: Relationship between childhood blood lead levels and stature. Pediatrics $1986 ; 77: 281-288$. 
24. Schwartz I., Otto D.: Blood lead level, hearing thresholds, and neuiobehavioral development in NHANES II children and youth. Cincinnati: Health Effects Research Laboratory, USEPA, 1986.

25. Rabinowitz M.B., Wetherill G.W., Kopple, J.D.: Kinetic analysis of lead metabolism in healthy humans. J Clin Invest 1976; 58: 260-270.

26. Mahaffey K.R. Nutritional factors in lead poisoning. Nutr Rev $1981 ; 39: 353 \cdot 362$.

27. Mahaffey K.R., Annest J.L., Roberts J., et al: National estimates of blood lead levels: United States 1976 - 1980: association with selected demographic and socioeconomic factors. N Engl J Med 1982; $307: 573-579$.

28. Mahaffey K.R., Goyer R.A. The influence of iron deficiency on tissue content and toxicity of ingested lead in the rat. 1 Lab Clin Med 1972; 79: 128-136.
29. Yip R., Schwartz S., Derinord A.S.: Screening for iron deficiency with the erythrocyte protoporphyrin test. Pediatrc 1983; 72: 214-219.

30. Piomelli $S$.' Free erythrocyte porphyrins in the detection of undue absorption of $\mathrm{Pb}$ and of $\mathrm{Fe}$ deficiency. Clin Chem 1977; 23: 264-269.

31. Mahoffey K.R., Annest J.L.: Association of erythrocyte protoporphyrin with blood lead level and iron status in the second National Health and $\mathrm{Nu}$ trition Examination Survey, 1976-1980. Environ Res $1986 ; 41: 327-338$.

32. Rios E., Olivares M., Amar $M$, et al.: Evaluation of iron status and prevalence of iron deficiency in infants in Chile. In Nurrition Intervention Strategies in National Development. Academic Press. 1983.

33. Anónimo. National Ambient Air Quality Standarớs. Environmental Protection Agency U.S.A. 1983. 